\title{
Effect of Laparoscopic Mini Gastric Bypass and Laparoscopic Sleeve Gastrectomy on Blood Glucose Level in Type 2 Diabetes Mellitus Obese Patients; a Prospective Study
}

\author{
Mohamed K.F. Hamed, Amr H. Afifi, Ahmed S. Emara \\ Department of General Surgery, Faculty of Medicine, Ain Shams University, Egypt
}

Background: Type 2 diabetes mellitus (T2DM) is now a global health problem. The World Health Organization estimates that between 2000 and 2030, the number of diabetic patients will increase by 114\%. T2DM and morbid obesity are conditions representing increasing public health threats. They are associated with significant morbidity and mortality. Despite lifestyle modifications and medical support, glycemic control remains difficult to achieve in obese diabetic patients.

Objective: To compare the effect of laparoscopic mini gastric bypass and laparoscopic sleeve gastrectomy in controlling blood glucose level in type 2 DM obese patients.

Patients and methods: This prospective study was conducted on 40 adult morbid obese patients with T2DM. Twenty patient underwent laparoscopic mini-gastric bypass and the other 20 patients underwent sleeve gastrectomy. All patients were followed up at 1, 3, 6, 9 and 12 months post-operatively by measuring BMI, excess weight-loss (EWL), Fasting and 2 hours post prandial blood glucose level, HbA1C also postoperative gastrograffin study to exclude complications.

Results: MGB was superior to SG in controlling T2DM along 1 year of follow up with lower 1 -year HBA1C, FBG, 2HPP blood glucose level.

Conclusion: MGB is safe and effective in controlling T2DM in morbidly obese patients candidate for bariatric surgery.

Key words: Type 2 diabetes mellitus, Mini-Gastric bypass, laparoscopic sleeve gastrectomy.

\section{Introduction}

Obesity is a major independent risk factor for the development of type 2 diabetes mellitus (T2DM) ${ }^{1}$

Studies showed that weight loss, even when modest, can reduce the incidence of T2DM in patients with impaired glucose tolerance, improve blood glucose control and other cardiovascular risk factors in patients with type 2 diabetic, while marked weight loss can even lead to resolution/ remission of diabetes. ${ }^{2}$

Pories et al. ${ }^{3}$ proposed that T2DM might be a surgical disease best treated via bariatric surgery.

Over the past 10 years, bariatric surgery has gained an increasing importance in the management of obese patients with T2DM. Moreover, the suggestion that the foregut plays an important role in the pathophysiology of T2DM opens up new possibilities for surgical approach in patients with T2DM, even in absence of severe obesity leading to a nominal shift from 'bariatric' to 'metabolic' surgery. ${ }^{4}$

A number of surgical approaches to induce weight loss have been developed, and many of them are also currently used in the management of obese patients with type 2 diabetes mellitus (T2DM). In general, these procedures can be classified as: solely restrictive [laparoscopic adjustable gastric banding (LAGB) and its variants, vertical banded gastroplasty (VGB) and laparoscopic sleeve gastrectomy], mostly restrictive [Roux-en-Y gastric bypass (RYGB)] and mostly malabsorptive [biliopancreatic diversion with duodenal switch (BPDS). ${ }^{5}$

These techniques proved to be effective in managing obese patients with or without T2DM. However, as these surgical procedures are different, their efficacy-to-safety balance also differ. Techniques with a component of malabsorption generally lead to more pronounced and more sustained weight loss compared to solely restrictive procedures. ${ }^{6}$

Early rapid weight loss and the percentage of excess weight lost were significant factors associated with diabetes remission. ${ }^{7}$

Factors considered during assessment of morbidly obese diabetic patient includes the preoperative 
insulin dose, HbA1c level, disease duration and presence of complications. The presence of complications (such as nephropathy and retinopathy) should probably be considered an indirect marker of longstanding, poorly controlled diabetes rather than a direct marker of surgical treatment failure. ${ }^{8}$

The risk and type of complications related to bariatric surgery among diabetic patients are not much different from those in a non-diabetic population, although some (such as infections) are more prevalent in diabetics. Surprisingly, bariatric surgery is remarkably safe in diabetic obese patients in spite of the large body size of patients, the frequency and seriousness of co-morbidities. ${ }^{9}$

The risk of hypoglycaemia sometimes reported in non-diabetic individuals late after RYGB does not appear to affect diabetic patients. However, caloric restriction and weight loss may dramatically improve glucose control and lead to early hypoglycaemia if no appropriate reduction in glucose-lowering therapies is made soon after surgery i.e. adjustment of medical treatment and caloric intake should be done to avoid hypoglycemia. ${ }^{10}$

The aim of this study was to compare the effect of laparoscopic mini gastric bypass and laparoscopic sleeve gastrectomy in controlling type 2 DM obese patients.

Study design: Forty (40) patients with BMI >35 with type 2 DM on oral hypoglycemic drugs (and not on insulin) were recruited in this prospective controlled study. They were randomly allocated into two equal groups; group (I) representing those who would undergo laparoscopic mini-gastric bypass and group (II) for those who would undergo laparoscopic sleeve gastrectomy. All patients were consented to participate in the study. An approval from Ain Shams University Hospitals' ethical Committee was obtained.

Inclusion criteria: Prospectively enrolled patients were within the age group $18-60$ years and body mass index (BMI) $>35 \mathrm{~kg} / \mathrm{m} 2$ presented with uncontrolled blood glucose level defined as: glycosylated hemoglobin A1c level more than or equal to $6.5 \%$, fasting plasma glucose level (FPG) more than or equal to 126 (fasting was defined as no caloric intake for at least 8 hours), a 2-hour plasma glucose more than or equal to $200 \mathrm{mg} / \mathrm{dl}$ during an oral glucose tolerance test (OGTT) or random blood glucose level more than or equal to $200 \mathrm{mg} /$ dl. Candidates were of accepted operative risk i.e. ASA I or I and psychologically stable (documented by psychiatric assessment at the psychiatry clinic of Ain Shams university hospitals), having a supportive family / social environment.
Patients with previous bariatric surgery and those with alcohol or substance abuse were excluded. Also, patients treated with insulin were excluded for the sake of avoiding bias in the results comparing the effect of surgical intervention on two different groups; one of them might have absolute, not relative, insulin insufficiency.

Procedure: Full clinical history including patient demographics, medical history, weight and patient dietary habits was taken from all participants followed by clinical examination including anthropometric measurements (height, weight, BMI). Preoperative blood tests (complete blood picture, coagulation profile, liver and kidney functions tests, lipids profile), pulmonary function tests and plain $x$ ray were done. Pelvi-abdominal ultrasound to exclude gall bladder stones was also done.

the procedure was done under general anesthesia, the patient was placed in supine position with the legs spread apart. The operating surgeon was positioned in between the patient's leg, the assisting surgeon on the left and camera assistance at the right side of the patient. After placement of a $1.5 \mathrm{~cm}$ supra-umbilical port via a transverse skin incision, pneumoperitoneum was attained with carbon dioxide gas under pressure of $12-15 \mathrm{mmHg}$. Two 12-mm trocars (one at about $10 \mathrm{~cm}$ below the xiphi-sternum to the left of the umbilicus while the other one at the right midclavicular line above the level of the umbilicus by $1 \mathrm{~cm}$ ) and three $5-\mathrm{mm}$ trocars (at the epigastium, at the left midclavicular line and at the anterior axillary line parallel to the left coatal margin) were used.

For mini-gastric bypass group i.e. group I, ligasure (LigaSure ${ }^{\circledR}$-Covidien, USA) was used to section the lesser omentum at the level of the crow's foot to enter the lesser sac.

An endoscopic stapler loaded with a 45-mm/3.5$\mathrm{mm}$ cartridge (Endo-GIA®-Covidien, USA) was inserted through the created opening (at the level of the incisura angularis in the lesser omentum) and applied, sectioning the stomach horizontally. A 36Fr orogastric tube (Ref $340.36 \AA$, Vygon, France) was inserted to calibrate the gastric reservoir. Fatty tissue and fibrous adhesions between the posterior gastric wall and pancreas were dissected. Then, an endoscopic stapler loaded with 60-mm/3.5$\mathrm{mm}$ cartridges (Endo-GIA®-Covidien, USA) was consecutively applied, sectioning the stomach vertically till the level of the left crus of the diaphragm completing the gastric reservoir.

After formation of the mini- gastric tube, the small intestine $200 \mathrm{~cm}$ distal from the ligament of Treitz was anastomosed with a mini-gastric tube in a sideto-side, antecolic, isoperistaltic fashion with a $30 \mathrm{~mm}$ 
endo-stapler (Endo GIA Universal Stapler, Covidien Autosuture, Mansfield, MA). The gastric and jejunalhole used for introducing the endo-stapler was closed with sutures (V Loc Suture, Ethicon Inc., Somerville, NJ). Consequently, the gastric antrum, duodenum, and proximal jejunum were bypassed. With the intention of foregut exclusion and bile reflux prevention, an anchoring suture was laid adjacent to the gastrojejunal anastomosis attaining acute angulation at the afferent limb, and simultaneously maintaining a parallel line between the long gastric tube and the efferent limb (Figures 1a,b).

For sleeve gastrectomy group i.e. group II, ligasure (LigaSure $\AA$-Covidien, USA) was used to section the greater omentum. The pylorus was identified. The starting point of future stapling was designed to be $2-8 \mathrm{~cm}$ proximal to the pylorus. The first window was created between the stomach and greater omentum at a slight proximal site. The distal stomach was grasped and pulled to the right and upper side and the greater omentum was spread to the counterdirection. Dissection continued proximally to get approach to short gastric vessels near the lower pole of the spleen. The short gastric vessels were divided near to the gastric wall carefully with least tension between the stomach and spleen.

The gastrophrenic ligament was incised to ensure complete mobilization of fundus. The dissection continued to the point of His angle and left diaphragmatic crus. A 36-Fr orogastric tube (Ref 340.36®, Vygon, France) was inserted to calibrate the gastric reservoir. Fatty tissue and fibrous adhesions between the posterior gastric wall and pancreas were dissected.

After full mobilization of the stomach, gastric division was performed using an endoscopic stapler loaded with 60-mm cartridges (Endo-GIA $®$-Covidien, USA) from the antrum toward the angle of His.

Stapling of the gastric antrum was performed with green cartridge (closed heights $2.0 \mathrm{~mm}, 4.8 / 60$ $\mathrm{mm}$ ) followed by sequential blue cartridges for the remaining corpus and fundus (closed height $1.5 \mathrm{~mm}$ 3.5/60 mm, blue). Compression for 10-20 s before each firing was performed. The stapler was fired consecutively along the bougie till the angle of His. During postoperative period, attention was paid to record body mass index (BMI), percentage of excess weight loss (\%EWL), HBA1c, Blood glucose level at the period of one month, three months, six months, nine months and twelve months postoperatively.

Complete patient remission was defined as stopping oral hypoglycemic drugs during the first-year interval after the operation while having a "within normal range" fasting, 2-hours post-prandial and HA1c blood glucose level. Consequently, partial remission was defined as sub-diabetic hyperglycemia for the same period of follow-up (1 year) without the need for pharmacologic therapy. Our reference values were those adopted by the "American Diabetes Association" i.e. HbA1c < 5.7\% for complete remission and $<6.5 \%$ for partial remission.

Statistical analysis: Recorded data were analyzed using the statistical package for social sciences, version 20.0 (SPSS Inc., Chicago, Illinois, USA). Quantitative data were expressed as mean \pm standard deviation (SD). Qualitative data were expressed as frequency and percentage.

The following tests were done: Independentsamples t-test of significance was used when comparing between two means. Chi-square (x2) test of significance was used in order to compare proportions between two qualitative parameters.

The confidence interval was set to $95 \%$ and the margin of error accepted was set to $5 \%$. So, the p-value was considered significant as the following: Probability (P-value). P-value $<0.05$ was considered significant. P-value $<0.001$ was considered as highly significant. P-value $>0.05$ was considered insignificant.

\section{Results}

In this study, we had two groups of morbidly obese patients with the mean age of 44.5 in both groups and demographic data as shown in Table 1. None of the recruited patients was smoker. The body mass index of both of the study groups is shown in Table 2 and Figures 5. The duration of diabetes of both groups is shown in Table $\mathbf{3}$ with no statistically significant difference between both groups.

There was a statistically significant difference in the percentage of excess weight loss (EWL \%) and percentage of diabetes remission favoring the minigastric bypass group i.e. group I (Tables $\mathbf{2 , 4 , 5}$, Figurs 5-7) which was evident by the mean value of FBG, 2HPP glucose and HbA1c at the postoperative follow-up visits at $1,3,6,9$ and 12 months (Tables 6-8 Figures 8-10).

A summary of the results of both groups is shown in Tables 9,10.

It is worth to state that our complication rate with MGB was low having 1 patient who developed reflux and another patient developed postoperative bleeding managed conservatively. In the SG group, only 1 patient out of 20 developed postoperative bleeding which was managed conservatively. All of the operations were completed laparoscopically without any conversions. 

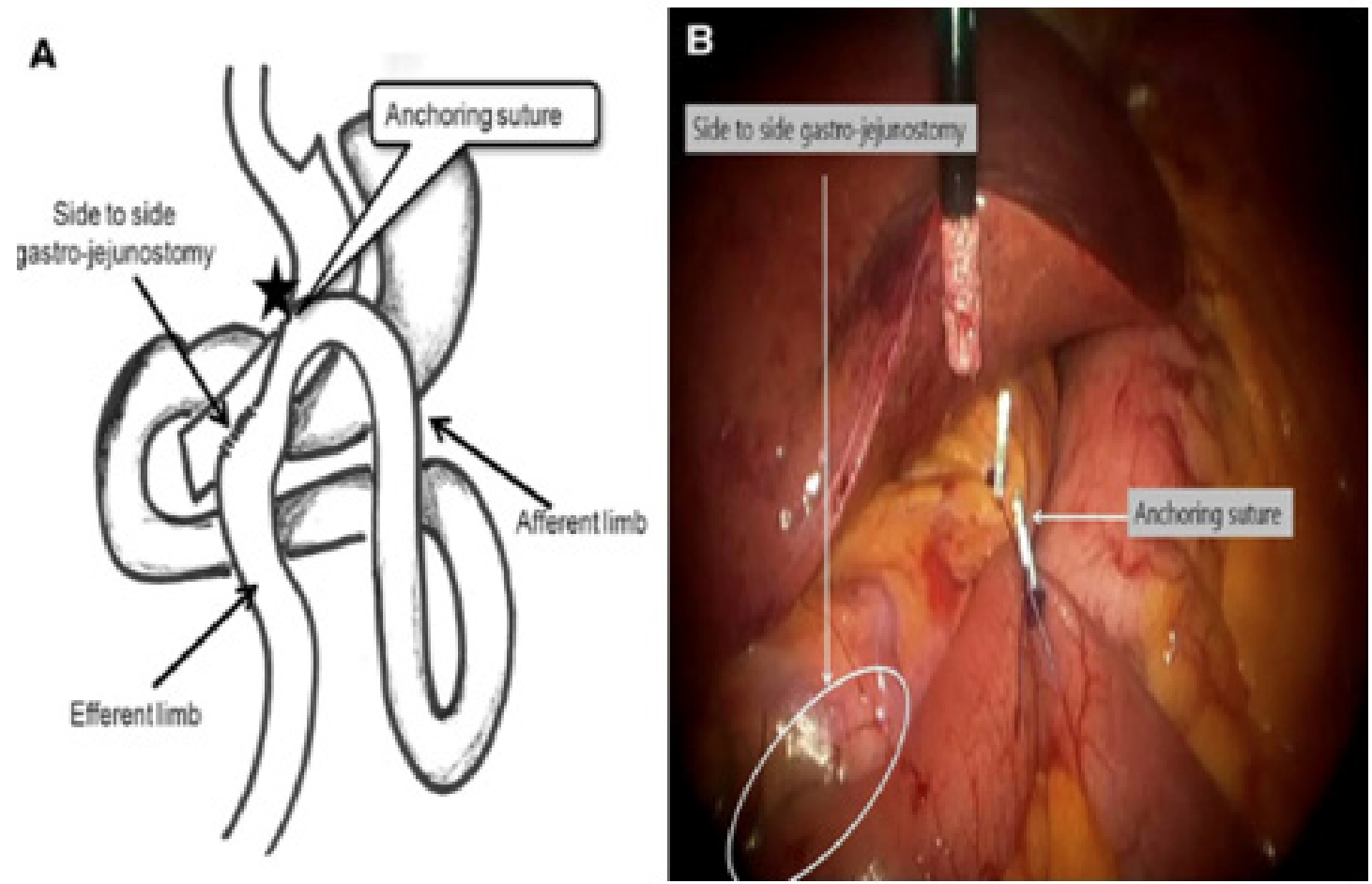

Fig 1: a) Diagrammatic illustration of laparoscopic mini-gastric bypass. An anchoring suture between the minigastric tube and the afferent limb was laid to attain an acute angulation at the afferent limb, and simultaneously, to maintain a parallel line between the long gastric tube and the efferent limb. b) Configuration of the gastrojejunal anastomosis. A steep angulation at the afferent limb was obtained with an anchoring suture adjacent to the anastomosis.

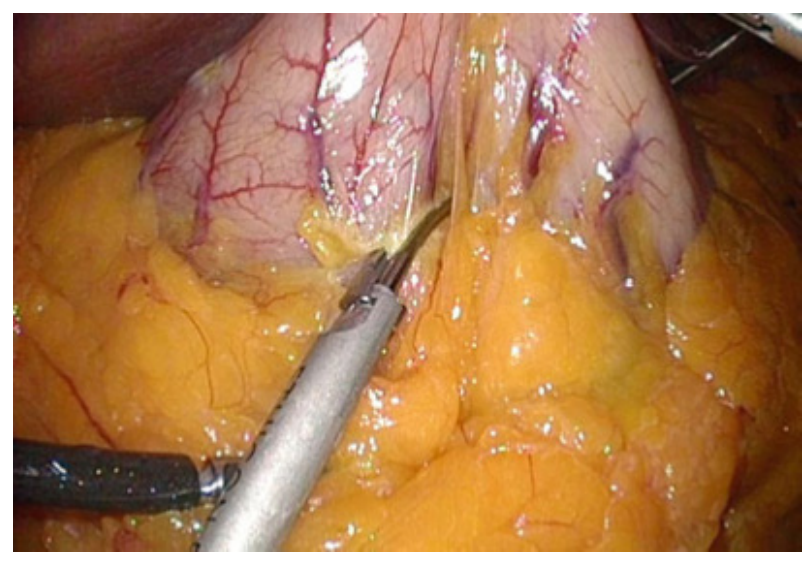

Fig 2: Dissection begins by making a window at a transparent area between arcade and it is easier to start dissection from the proximal to the marking point.

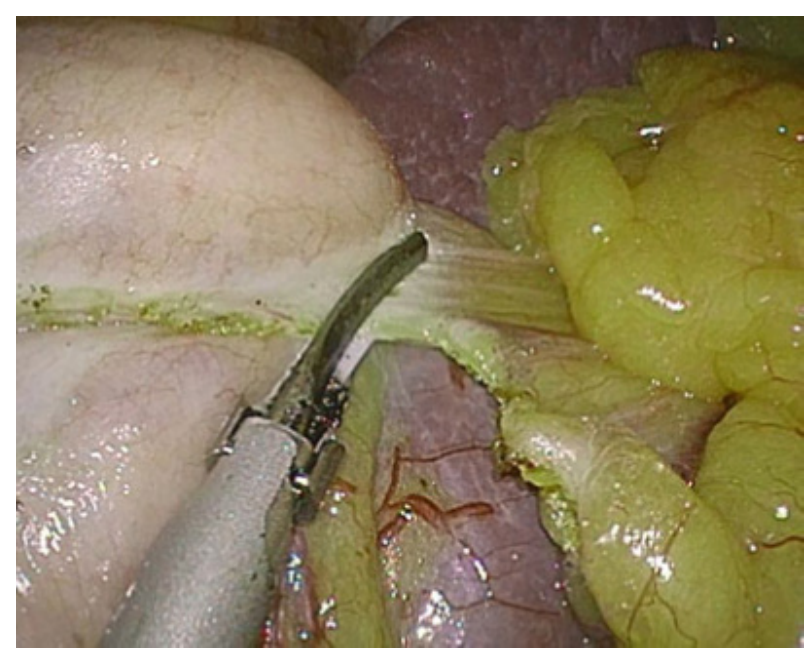

Fig 3: Short gastric vessel division close to gastric wall. Tension between the fundus and spleen should be minimized to prevent splenic tear. 


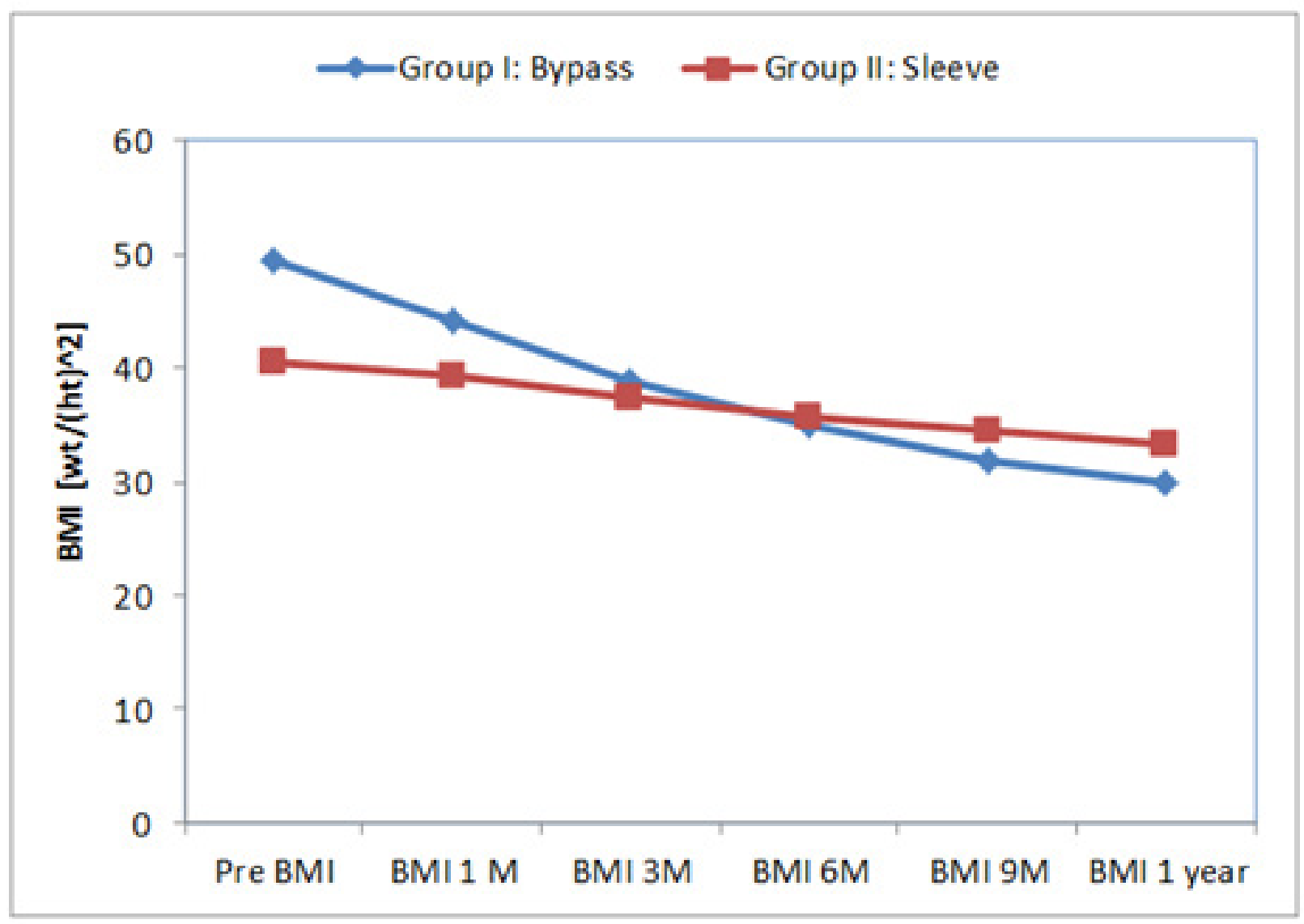

Fig 5: Shows line chart between groups according to BMI.

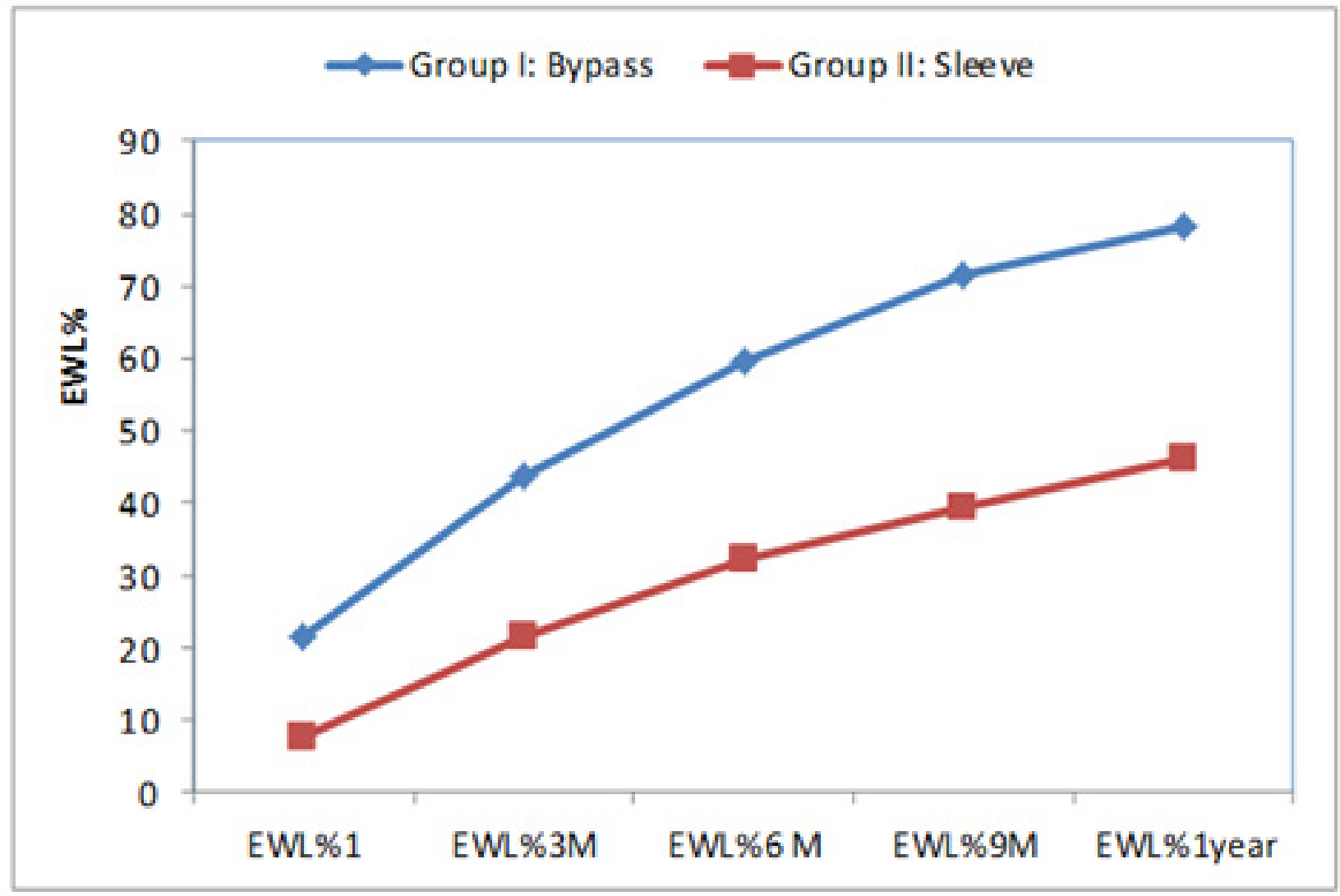

Fig 6: Shows line chart between groups according to EWL\%. 


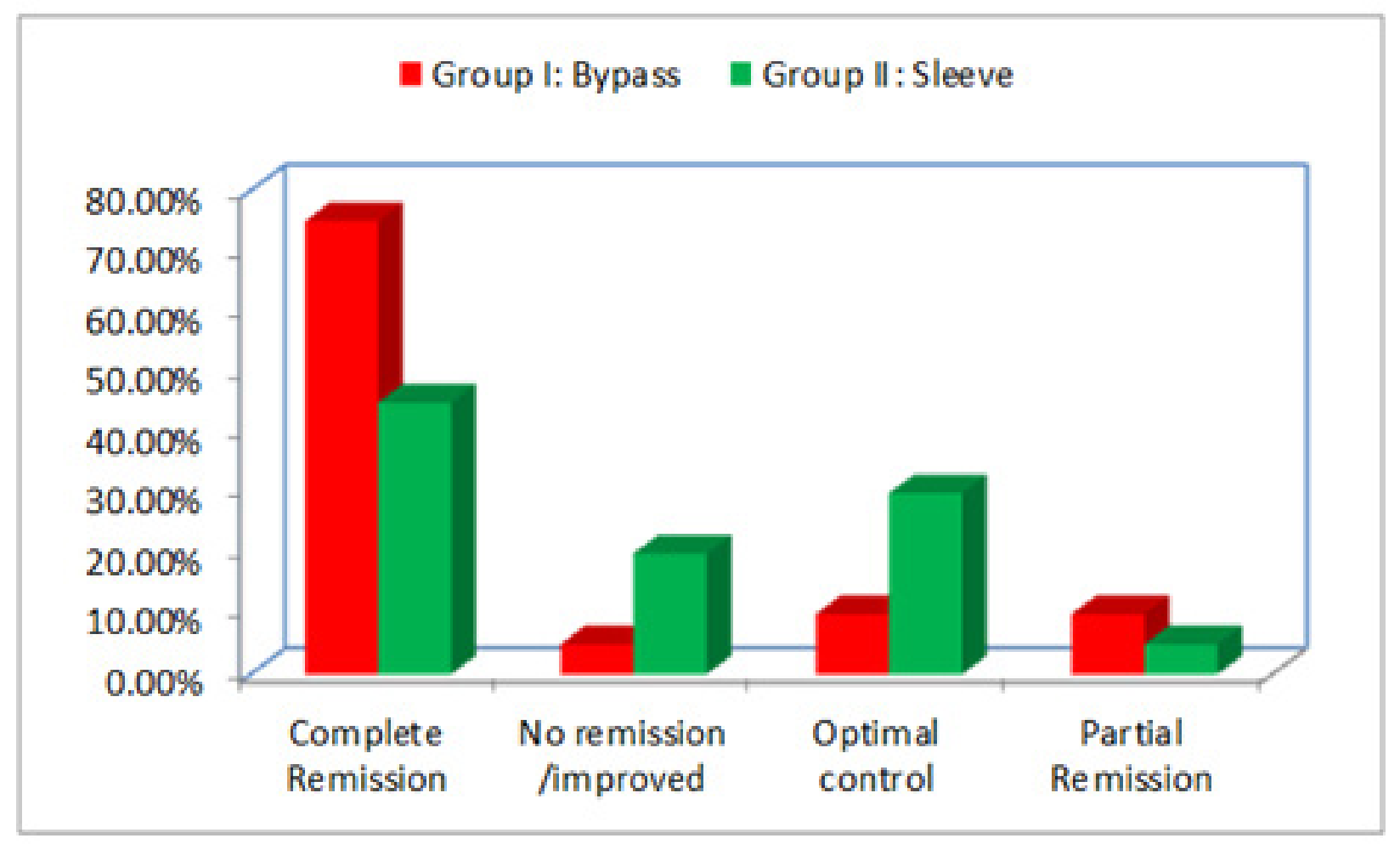

Fig 7: Bar chart between groups according to remission.

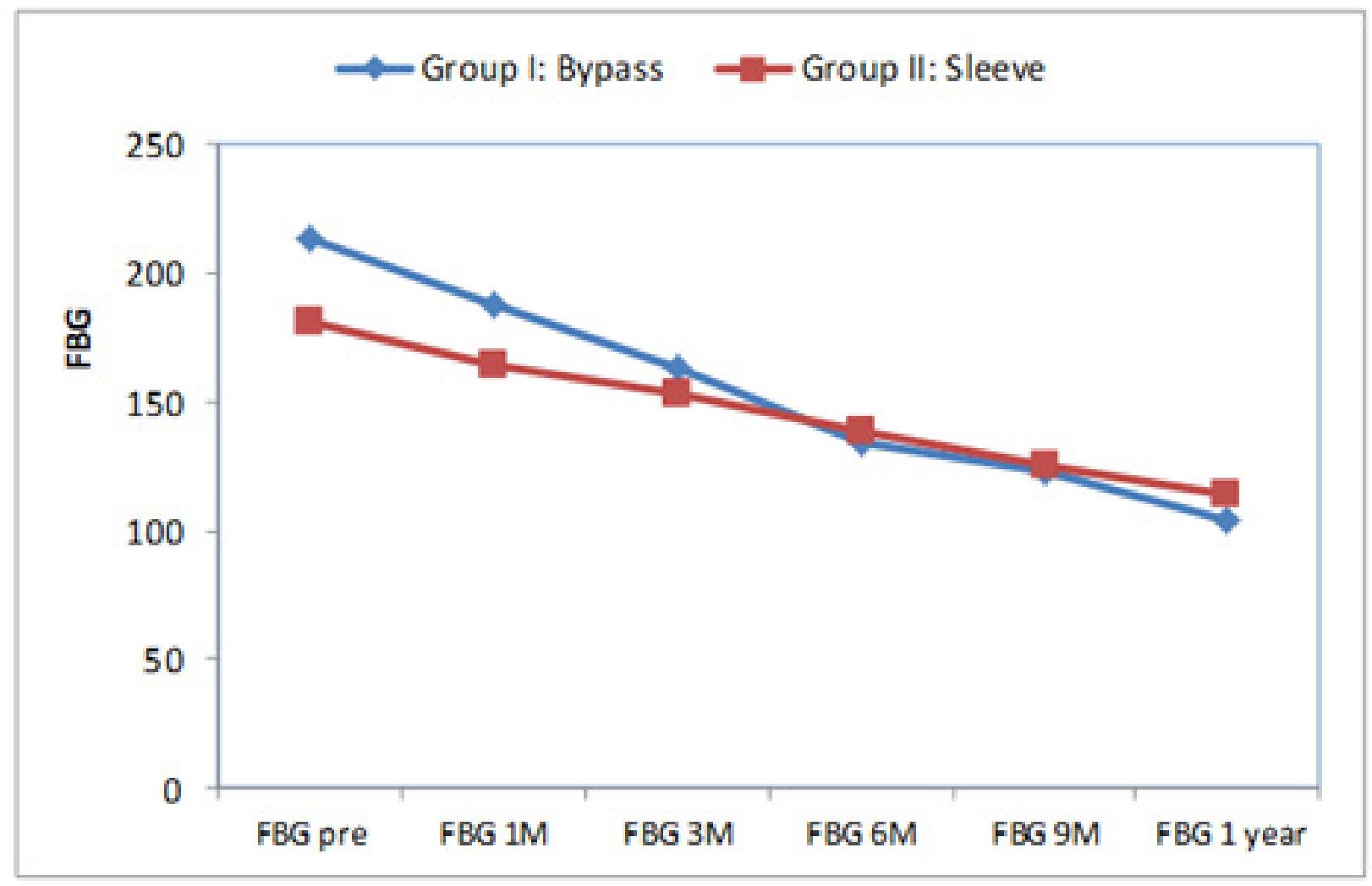

Fig 8: Shows line chart between groups according to FBG. 


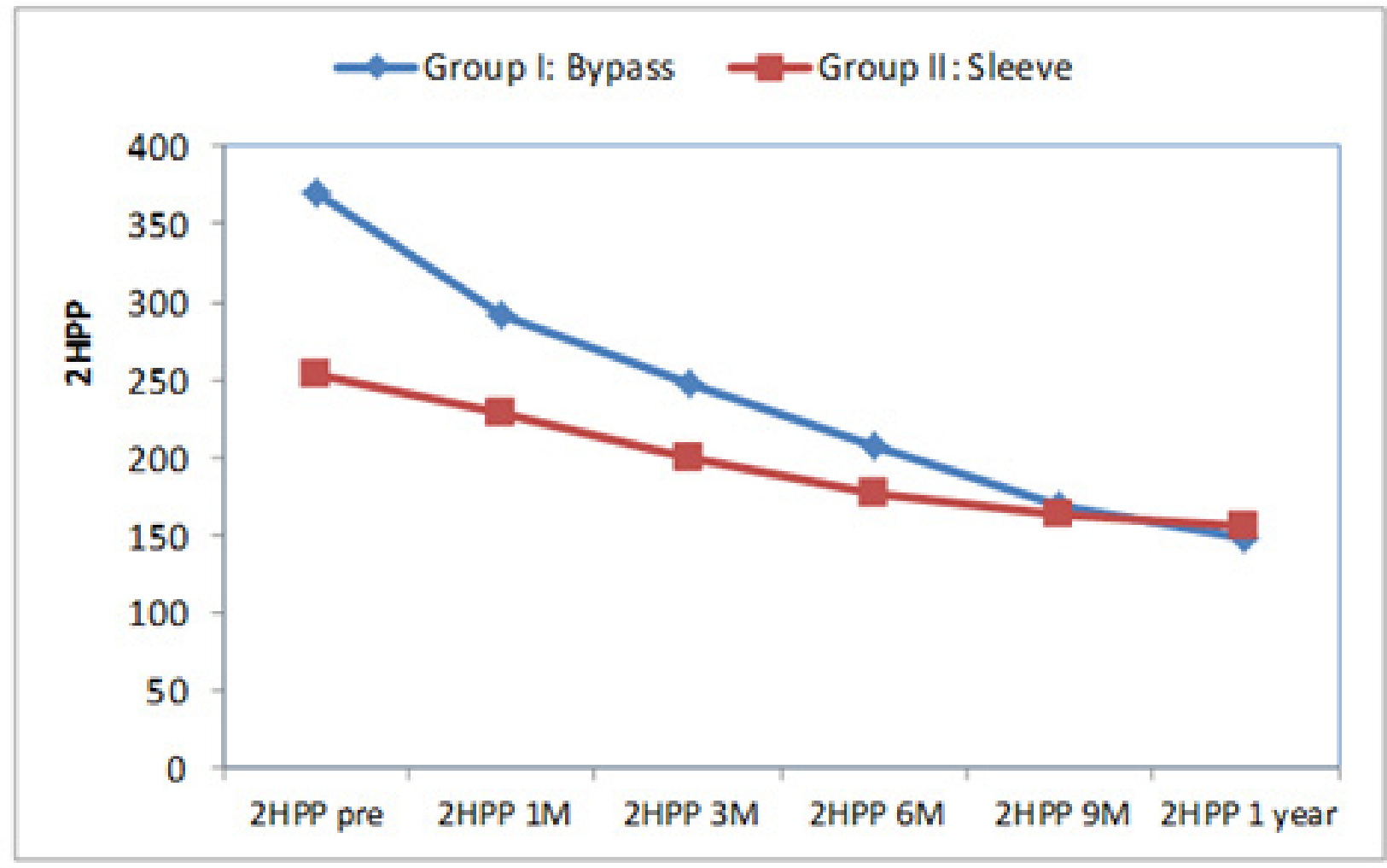

Fig 9: Shows line chart between groups according to $2 \mathrm{HPP}$.

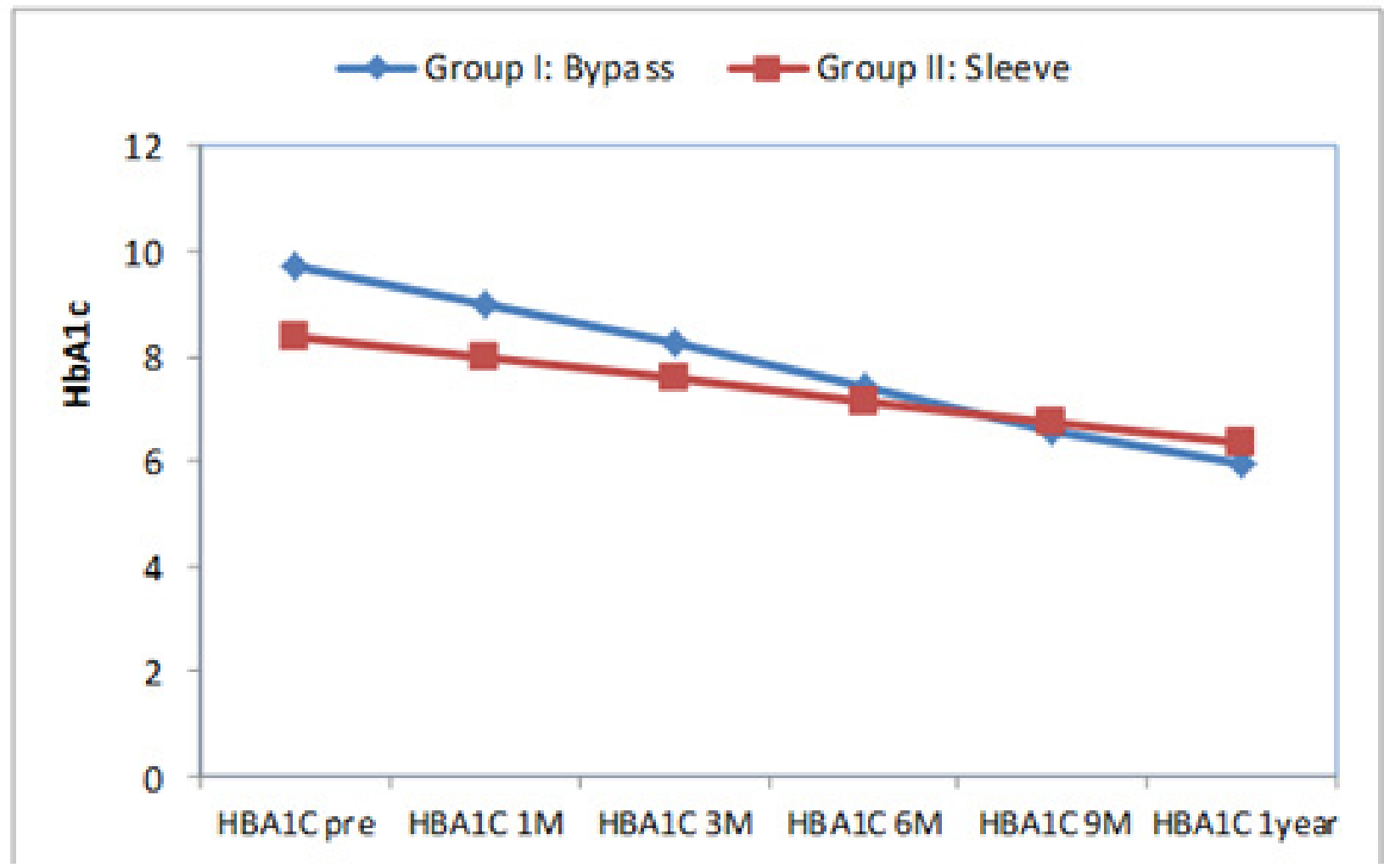

Fig 10: Shows line chart between groups according to HbA1c. 
Table 1: Comparison between groups according to demographic data

\begin{tabular}{|c|c|c|c|c|}
\hline Demographic Data & $\begin{array}{l}\text { Group I: Bypass } \\
(N=20)\end{array}$ & $\begin{array}{l}\text { Group II: Sleeve } \\
\qquad(N=20)\end{array}$ & $t / \times 2 \#$ & P-value \\
\hline \multicolumn{5}{|l|}{ Age (years) } \\
\hline Mean \pm SD & $44.55 \pm 4.93$ & $44.65 \pm 4.57$ & \multirow{2}{*}{-0.067} & \multirow{2}{*}{0.947} \\
\hline Range & $36-52$ & $37-52$ & & \\
\hline \multicolumn{5}{|l|}{ Sex } \\
\hline Male & $9(45.0 \%)$ & $8(40.0 \%)$ & \multirow{2}{*}{$0.102 \#$} & \multirow{2}{*}{0.749} \\
\hline Female & $11(55.0 \%)$ & $12(60.0 \%)$ & & \\
\hline
\end{tabular}

t-Independent Sample t-test; \#x2: Chi-square test.

p-value $>0.05$ NS.

Table 2: Comparison between groups according to BMI

\begin{tabular}{|c|c|c|c|c|}
\hline BMI [wt/(ht)^2] & $\begin{array}{l}\text { Group I: By- } \\
\text { pass }(\mathbf{N}=\mathbf{2 0})\end{array}$ & $\begin{array}{c}\text { Group II: } \\
\text { Sleeve }(\mathrm{N}=\mathbf{2 0})\end{array}$ & t-test & P-value \\
\hline \multicolumn{5}{|l|}{ Pre BMI } \\
\hline Mean \pm SD & $49.55 \pm 3.68$ & $40.55 \pm 3.14$ & \multirow{2}{*}{8.327} & \multirow{2}{*}{$<0.001 * *$} \\
\hline Range & $42-56$ & $36-46$ & & \\
\hline \multicolumn{5}{|l|}{ BMI 1 M } \\
\hline Mean \pm SD & $44.25 \pm 3.18$ & $39.38 \pm 2.93$ & \multirow{2}{*}{5.042} & \multirow{2}{*}{$<0.001 * *$} \\
\hline Range & $38-49$ & $35-44.5$ & & \\
\hline \multicolumn{5}{|l|}{ BMI 3M } \\
\hline Mean \pm SD & $38.90 \pm 2.00$ & $37.28 \pm 2.61$ & \multirow{2}{*}{1.210} & \multirow{2}{*}{0.133} \\
\hline Range & $34-42$ & $33-42$ & & \\
\hline \multicolumn{5}{|l|}{ BMI 6M } \\
\hline Mean $\pm S D$ & $35.05 \pm 1.43$ & $35.58 \pm 2.34$ & \multirow{2}{*}{-0.857} & \multirow{2}{*}{0.397} \\
\hline Range & $31-37$ & $31-39$ & & \\
\hline \multicolumn{5}{|l|}{ BMI 9M } \\
\hline Mean \pm SD & $31.88 \pm 1.30$ & $34.40 \pm 2.02$ & \multirow{2}{*}{-4.709} & \multirow{2}{*}{$<0.001^{* *}$} \\
\hline Range & $29-34$ & $30.5-38$ & & \\
\hline \multicolumn{5}{|l|}{ BMI 1 year } \\
\hline Mean \pm SD & $29.80 \pm 1.23$ & $33.38 \pm 1.86$ & \multirow{2}{*}{-7.187} & \multirow{2}{*}{$<0.001^{* *}$} \\
\hline Range & $\mathrm{s}$ & $30-37$ & & \\
\hline
\end{tabular}

t-Independent Sample t-test; $* *$ p-value $<0.001 \mathrm{HS}$.

This table shows statistically significant difference between groups according to BMI pre, after $1 \mathrm{~m}, 9 \mathrm{~m}$ and after 1 year.

Table 3: Comparison between groups according to duration of DM (years)

\begin{tabular}{|c|c|c|c|c|}
\hline Duration of DM (years) & $\begin{array}{c}\text { Group I: Bypass } \\
(\mathbf{N}=\mathbf{2 0})\end{array}$ & $\begin{array}{l}\text { Group II: Sleeve } \\
(\mathbf{N}=\mathbf{2 0})\end{array}$ & t-test & p-value \\
\hline Mean \pm SD & $5.88 \pm 2.80$ & $6.85 \pm 2.57$ & \multirow{2}{*}{-1.148} & \multirow{2}{*}{0.258} \\
\hline Range & $2-12$ & $2-11$ & & \\
\hline
\end{tabular}

t-Independent Sample t-test; p-value >0.05 NS. 
Table 4: Comparison between groups according to EWL\%

\begin{tabular}{|c|c|c|c|c|}
\hline EWL\% & Group I: Bypass $(\mathrm{N}=20)$ & Group II: Sleeve $(\mathrm{N}=20)$ & t-test & P-value \\
\hline \multicolumn{5}{|l|}{ EWL\%1M } \\
\hline Mean \pm SD & $21.53 \pm 3.41$ & $7.56 \pm 1.76$ & \multirow{2}{*}{16.294} & \multirow{2}{*}{$<0.001^{* *}$} \\
\hline Range & $16-28.5$ & $5-12.5$ & & \\
\hline \multicolumn{5}{|l|}{ EWL\%3M } \\
\hline Mean \pm SD & $43.68 \pm 3.60$ & $21.44 \pm 3.76$ & \multirow{2}{*}{19.103} & \multirow{2}{*}{$<0.001^{* *}$} \\
\hline Range & $35-50$ & $14-27$ & & \\
\hline \multicolumn{5}{|l|}{ EWL\%6 M } \\
\hline Mean \pm SD & $59.54 \pm 4.95$ & $32.13 \pm 4.41$ & \multirow{2}{*}{0.983} & \multirow{2}{*}{0.332} \\
\hline Range & $52-70$ & $27.7-45.4$ & & \\
\hline \multicolumn{5}{|l|}{ EWL\%9M } \\
\hline Mean \pm SD & $71.69 \pm 2.68$ & $39.38 \pm 3.66$ & \multirow{2}{*}{31.872} & \multirow{2}{*}{$<0.001 * *$} \\
\hline Range & $66-76$ & $35-50$ & & \\
\hline \multicolumn{5}{|l|}{ EWL\%1year } \\
\hline Mean \pm SD & $78.27 \pm 3.26$ & $46.21 \pm 3.00$ & \multirow{2}{*}{32.359} & \multirow{2}{*}{$<0.001 * *$} \\
\hline Range & $70.8-83.8$ & $41-54.5$ & & \\
\hline
\end{tabular}

t-Independent Sample t-test; $* *$ p-value $<0.001 \mathrm{HS}$.

Table 5: Comparison between groups according to remission

\begin{tabular}{|c|c|c|c|c|}
\hline Remission & $\begin{array}{l}\text { Group I: By- } \\
\text { pass }(N=20)\end{array}$ & $\begin{array}{c}\text { Group II: } \\
\text { Sleeve } \\
(\mathbf{N}=20)\end{array}$ & $\mathbf{x 2}$ & P-value \\
\hline Complete Remission & $15(75.0 \%)$ & $9(45.0 \%)$ & \multirow{4}{*}{8.633} & \multirow{4}{*}{ 0.013* } \\
\hline Partial Remission & $2(10.0 \%)$ & $1(5.0 \%)$ & & \\
\hline Optimal control & $2(10.0 \%)$ & $6(30.0 \%)$ & & \\
\hline No remission /improved & $1(5.0 \%)$ & $4(20.0 \%)$ & & \\
\hline
\end{tabular}

x2: Chi-square test; ${ }^{*}$ p-value $<0.05 \mathrm{~S}$.

Table 6: Comparison between groups according to FBG

\begin{tabular}{|c|c|c|c|c|}
\hline FBG & Group I: Bypass $(\mathrm{N}=20)$ & Group II: Sleeve $(\mathbf{N}=\mathbf{2 0})$ & t-test & P-value \\
\hline \multicolumn{5}{|l|}{ FBG pre } \\
\hline Mean $\pm S D$ & $213.60 \pm 22.73$ & $181.15 \pm 41.86$ & \multirow{2}{*}{3.047} & \multirow{2}{*}{ 0.004* } \\
\hline Range & $185-269$ & $132-252$ & & \\
\hline \multicolumn{5}{|l|}{ FBG $1 M$} \\
\hline Mean \pm SD & $187.65 \pm 17.48$ & $164.75 \pm 32.22$ & \multirow{2}{*}{2.794} & \multirow{2}{*}{$0.008^{*}$} \\
\hline Range & $158-212$ & $128-220$ & & \\
\hline \multicolumn{5}{|l|}{ FBG $3 M$} \\
\hline Mean \pm SD & $163.30 \pm 16.67$ & $153.25 \pm 24.98$ & \multirow{2}{*}{1.497} & \multirow{2}{*}{0.143} \\
\hline Range & $139-189$ & $125-192$ & & \\
\hline \multicolumn{5}{|l|}{ FBG 6M } \\
\hline Mean $\pm S D$ & $134.50 \pm 10.70$ & $138.40 \pm 14.30$ & \multirow{2}{*}{-0.977} & \multirow{2}{*}{0.335} \\
\hline Range & $118-153$ & $119-167$ & & \\
\hline \multicolumn{5}{|l|}{ FBG 9M } \\
\hline Mean \pm SD & $123.45 \pm 7.70$ & $125.10 \pm 11.19$ & \multirow{2}{*}{-0.543} & \multirow{2}{*}{0.590} \\
\hline Range & $111-140$ & $110-148$ & & \\
\hline \multicolumn{5}{|c|}{ FBG 1 year } \\
\hline Mean \pm SD & $104.50 \pm 14.21$ & $113.75 \pm 14.49$ & \multirow{2}{*}{-2.038} & \multirow{2}{*}{$0.049 *$} \\
\hline Range & $93-138$ & $95-138$ & & \\
\hline
\end{tabular}

t-Independent Sample t-test; *p-value $<0.05 \mathrm{~S}$.

This table shows statistically significant difference between groups according to FBG pre, after $1 \mathrm{~m}$ and after 1 year. 
Table 7: Comparison between groups according to 2HPP

\begin{tabular}{|c|c|c|c|c|}
\hline 2HPP & $\begin{array}{l}\text { Group I: Bypass } \\
(\mathrm{N}=\mathbf{2 0})\end{array}$ & $\begin{array}{c}\text { Group II: Sleeve } \\
(\mathrm{N}=\mathbf{2 0})\end{array}$ & t-test & P-value \\
\hline \multicolumn{5}{|l|}{ 2HPP pre } \\
\hline Mean $\pm S D$ & $369.30 \pm 32.60$ & $253.40 \pm 32.91$ & \multirow{2}{*}{11.190} & \multirow{2}{*}{$<0.001^{* *}$} \\
\hline Range & $313-420$ & $210-316$ & & \\
\hline \multicolumn{5}{|l|}{ 2HPP 1M } \\
\hline Mean \pm SD & $291.90 \pm 11.39$ & $227.40 \pm 31.89$ & \multirow{2}{*}{8.519} & \multirow{2}{*}{$<0.001 * *$} \\
\hline Range & $265-310$ & $193-301$ & & \\
\hline \multicolumn{5}{|l|}{ 2HPP 3M } \\
\hline Mean $\pm S D$ & $248.05 \pm 19.46$ & $200.20 \pm 27.72$ & \multirow{2}{*}{6.318} & \multirow{2}{*}{$<0.001^{* *}$} \\
\hline Range & $215-280$ & $170-266$ & & \\
\hline \multicolumn{5}{|l|}{ 2HPP 6M } \\
\hline Mean $\pm S D$ & $206.65 \pm 17.19$ & $176.00 \pm 28.15$ & \multirow{2}{*}{4.156} & \multirow{2}{*}{$<0.001^{* *}$} \\
\hline Range & $180-260$ & $141-239$ & & \\
\hline \multicolumn{5}{|l|}{ 2HPP 9M } \\
\hline Mean \pm SD & $169.20 \pm 19.92$ & $161.95 \pm 27.30$ & \multirow{2}{*}{0.959} & \multirow{2}{*}{0.343} \\
\hline Range & $152-230$ & $138-221$ & & \\
\hline \multicolumn{5}{|c|}{ 2HPP 1 year } \\
\hline Mean $\pm S D$ & $146.70 \pm 21.87$ & $154.35 \pm 26.48$ & \multirow{2}{*}{-0.996} & \multirow{2}{*}{0.326} \\
\hline Range & $132-208$ & $133-210$ & & \\
\hline
\end{tabular}

t-Independent Sample t-test; $* *$ p-value $<0.001 \mathrm{HS}$.

This table shows statistically significant difference between groups according to 2HPP pre, after $1 \mathrm{~m}, 3 \mathrm{~m}$ and after $6 \mathrm{~m}$.

Table 8: Comparison between groups according to HbA1c

\begin{tabular}{|c|c|c|c|c|}
\hline HbA1c & $\begin{array}{l}\text { Group I: Bypass } \\
(\mathrm{N}=\mathbf{2 0 )}\end{array}$ & $\begin{array}{l}\text { Group II: Sleeve } \\
\qquad(\mathrm{N}=\mathbf{2 0})\end{array}$ & t-test & P-value \\
\hline \multicolumn{5}{|c|}{ HBA1C pre } \\
\hline Mean $\pm S D$ & $9.70 \pm 0.84$ & $8.35 \pm 1.09$ & \multirow{2}{*}{4.385} & \multirow{2}{*}{$<0.001^{* *}$} \\
\hline Range & $8.6-12$ & $6.4-10.5$ & & \\
\hline \multicolumn{5}{|c|}{ HBA1C 1M } \\
\hline Mean $\pm S D$ & $8.99 \pm 0.68$ & $7.97 \pm 0.88$ & \multirow{2}{*}{4.130} & \multirow{2}{*}{$<0.001^{* *}$} \\
\hline Range & $7.9-10.5$ & $6.4-10$ & & \\
\hline \multicolumn{5}{|l|}{ HBA1C 3M } \\
\hline Mean \pm SD & $8.24 \pm 0.49$ & $7.56 \pm 0.80$ & \multirow{2}{*}{3.222} & \multirow{2}{*}{$0.003^{*}$} \\
\hline Range & 7.1-9.4 & $6.2-9.6$ & & \\
\hline \multicolumn{5}{|c|}{ HBA1C 6M } \\
\hline Mean $\pm S D$ & $7.42 \pm 0.38$ & $7.12 \pm 0.69$ & \multirow{2}{*}{2.694} & \multirow{2}{*}{0.109} \\
\hline Range & $6.8-8$ & $6-9$ & & \\
\hline \multicolumn{5}{|c|}{ HBA1C 9M } \\
\hline Mean \pm SD & $6.60 \pm 0.42$ & $6.73 \pm 0.67$ & \multirow{2}{*}{-0.735} & \multirow{2}{*}{0.467} \\
\hline Range & $6.1-7.9$ & $6-8.6$ & & \\
\hline \multicolumn{5}{|c|}{ HBA1C 1year } \\
\hline Mean \pm SD & $5.93 \pm 0.60$ & $6.33 \pm 0.82$ & \multirow{2}{*}{-1.769} & \multirow{2}{*}{0.085} \\
\hline Range & $5.4-7.7$ & $5.4-8.1$ & & \\
\hline
\end{tabular}

t-Independent Sample t-test; *p-value $<0.05 \mathrm{~S} ; * *$ p-value $<0.001 \mathrm{HS}$.

This table shows statistically significant difference between groups according to HbA1c pre, after $1 \mathrm{~m}$ and after $3 \mathrm{~m}$. 
Table 9: Relation between remission with age, FBG, 2HPP, BMI, EWL\%, HBA1C and duration of DM (years) in group I bypass

\begin{tabular}{|c|c|c|c|c|c|c|c|}
\hline Group I: Bypass & & $\begin{array}{l}\text { Com- } \\
\text { plete } \\
\text { Remis- } \\
\text { sion }\end{array}$ & $\begin{array}{l}\text { Partial } \\
\text { Remis- } \\
\text { sion }\end{array}$ & $\begin{array}{c}\text { Opti- } \\
\text { mal } \\
\text { control }\end{array}$ & $\begin{array}{l}\text { No re- } \\
\text { mission / } \\
\text { improved }\end{array}$ & ANOVA & P-value \\
\hline \multirow{2}{*}{ Age (years) } & Mean & 45.67 & 46.50 & 37.50 & 38.00 & \multirow{2}{*}{3.076} & \multirow{2}{*}{0.058} \\
\hline & \pm SD & 4.50 & 2.12 & 2.12 & 0.00 & & \\
\hline \multirow{2}{*}{ FBG pre } & Mean & 209.87 & 211.00 & 239.50 & 223.00 & \multirow{2}{*}{1.080} & \multirow{2}{*}{0.386} \\
\hline & $\pm \mathbf{S D}$ & 21.42 & 0.00 & 41.72 & 0.00 & & \\
\hline \multirow{2}{*}{ 2HPP pre } & Mean & 359.13 & 388.00 & 409.00 & 405.00 & \multirow{2}{*}{2.635} & \multirow{2}{*}{0.085} \\
\hline & $\pm S D$ & 30.79 & 0.00 & 15.56 & 0.00 & & \\
\hline \multirow{2}{*}{ Pre BMI } & Mean & 48.80 & 50.00 & 53.50 & 52.00 & \multirow{2}{*}{1.165} & \multirow{2}{*}{0.354} \\
\hline & \pm SD & 3.76 & 0.00 & 3.54 & 0.00 & & \\
\hline \multirow{2}{*}{ EWL\%1year } & Mean & 78.19 & 78.00 & 77.50 & 81.40 & \multirow{2}{*}{0.313} & \multirow{2}{*}{0.816} \\
\hline & \pm SD & 3.49 & 2.83 & 3.54 & 0.00 & & \\
\hline \multirow{2}{*}{ HBA1C pre } & Mean & 9.47 & 9.50 & 10.95 & 11.00 & \multirow{2}{*}{3.916} & \multirow{2}{*}{$0.028^{*}$} \\
\hline & \pm ISD & 0.63 & 0.00 & 1.48 & 0.00 & & \\
\hline \multirow[t]{2}{*}{ Duration of DM (years) } & Mean & 4.57 & 9.00 & 10.50 & 10.00 & \multirow[b]{2}{*}{12.894} & \multirow[b]{2}{*}{$<0.001^{* *}$} \\
\hline & $\pm S D$ & 1.62 & 1.41 & 2.12 & 0.00 & & \\
\hline
\end{tabular}

This table shows statistically significant relation between remission with HBA1C pre and duration of DM (years).

Table 10: Relation between remission with age, FBG, 2HPP, BMI, EWL\%, HBA1C and duration of DM (years) in group II sleeve

\begin{tabular}{|c|c|c|c|c|c|c|c|}
\hline Group II: Sleeve & & $\begin{array}{c}\text { Complete } \\
\text { Remis- } \\
\text { sion }\end{array}$ & $\begin{array}{c}\text { Partial } \\
\text { Remis- } \\
\text { sion }\end{array}$ & $\begin{array}{c}\text { Opti- } \\
\text { mal } \\
\text { control }\end{array}$ & $\begin{array}{c}\text { No re- } \\
\text { mission / } \\
\text { improved }\end{array}$ & ANOVA & P-value \\
\hline \multirow{2}{*}{ Age (years) } & Mean & 45.78 & 49.00 & 41.50 & 45.75 & \multirow{2}{*}{1.674} & \multirow{2}{*}{0.212} \\
\hline & $\pm S D$ & 4.27 & 0.00 & 4.76 & 3.77 & & \\
\hline \multirow[t]{2}{*}{ FBG pre } & Mean & 159.89 & 137.00 & 195.00 & 219.25 & \multirow[b]{2}{*}{3.406} & \multirow[b]{2}{*}{$0.043^{*}$} \\
\hline & \pm SD & 23.85 & 0.00 & 45.62 & 42.30 & & \\
\hline \multirow{2}{*}{ 2HPP pre } & Mean & 239.00 & 246.00 & 246.00 & 298.75 & \multirow{2}{*}{5.534} & \multirow{2}{*}{0.008} \\
\hline & \pm SD & 24.45 & 0.00 & 28.72 & 19.92 & & \\
\hline \multirow{2}{*}{ Pre BMI } & Mean & 40.33 & 42.00 & 39.83 & 41.75 & \multirow{2}{*}{0.345} & \multirow{2}{*}{0.793} \\
\hline & \pm SD & 3.35 & 0.00 & 1.60 & 4.92 & & \\
\hline \multirow{2}{*}{ EWL\%1year } & Mean & 46.56 & 47.00 & 45.43 & 46.40 & \multirow{2}{*}{0.176} & \multirow{2}{*}{0.911} \\
\hline & $\pm S D$ & 3.95 & 0.00 & 1.13 & 3.36 & & \\
\hline \multirow{2}{*}{ HBA1C pre } & Mean & 7.70 & 7.80 & 8.37 & 9.90 & \multirow{2}{*}{8.300} & \multirow{2}{*}{$<0.001 * *$} \\
\hline & \pm SD & 0.65 & 0.00 & 0.94 & 0.59 & & \\
\hline Duration of & Mean & 4.50 & 7.00 & 8.83 & 9.13 & \multirow{2}{*}{15.910} & \multirow{2}{*}{$<0.001 * *$} \\
\hline DM (years) & $\pm \mathbf{S D}$ & 1.50 & 0.00 & 1.51 & 0.85 & & \\
\hline
\end{tabular}

This table shows statistically significant relation between remission with FBG, HBA1C pre and duration of DM (years). 


\section{Discussion}

Roux-en-Y gastric bypass (RYGB) and sleeve gastrectomy (SG) rank among the most frequently applied bariatric procedures worldwide due to their positive benefit/risk correlation. Bypass procedures are suggested to be more effective treating diabetes mellitus than other procedures including sleeve gastrectomy and are followed by normalization of concentrations of plasma glucose and $\mathrm{HbA1c}$ in 80 $100 \%$ of morbidly obese patients. ${ }^{8}$

Although the standard laparoscopic Roux-en-Y gastric bypass (LRYGB) is accepted as the gold standard of bariatric surgery and diabetes remission, it carries a high complication rate in comparison to other procedures. Laparoscopic mini-gastric bypass is reported to be a safe alternative to LRYGB, with similar efficacy in weight reduction and resolution of metabolic complications, including diabetes. ${ }^{11}$

Small trials have shown glycemic benefit of bariatric surgery in patients with type 2 diabetes and BMI of $30-35 \mathrm{~kg} / \mathrm{m} 2$. However, there is currently insufficient evidence to generally recommend surgery in patients with BMI more than $35 \mathrm{~kg} / \mathrm{m} 2$ outside of a research protocol. (1) Moreover, the definition of remission of T2DM after bariatric surgery is still widely debated. ${ }^{12}$

In our study, the mean age group was nearly the same in both groups (44.5) (Table 1). Our study showed no significant relationship between age and T2DM remission (Tables $\mathbf{9 , 1 0}$ ). This could be attributed to the small sample size and common age group used in both MGB and SG groups. This does not meet with studies made by Still et al. ${ }^{13}$ and Huang et al. ${ }^{14}$

Still et al. ${ }^{13}$ studied 690 patients and observed that each 10-year decrease in age was associated with a greater odds ratio (OR) of early (1.41 [1.10-1.80], $\mathrm{p}=0.0071)$ and later remission of T2DM (1.45 [1.101.92], $p=0.0085)$.

Similar findings have also been observed by Huang et al. ${ }^{14}$ in a study of 22 Chinese patients with a BMI between $25-35 \mathrm{~kg} / \mathrm{m} 2$.

Furthermore, a German study by Jurowich et al. ${ }^{15}$ found that increasing age was an independent significant predictor for postoperative failure of diabetes remission.

In our study, the mean duration of DM was 5.8 ranging from 2-12 years in mini gastric bypass group while the mean duration of DM was 6.8 ranging from 2-11 years in sleeve gastrectomy group (Table $\mathbf{3}$ ). There was a significant difference in response between patients who were recently diagnosed with diabetes and patients with longer time diagnosis of diabetes mellitus finding higher remission rate in recently diagnosed T2DM patients ( $p$ value $<0.001$ ) (Tables 9,10). This meets with the studies conducted by Pories et al. ${ }^{16}$ and Schauer. ${ }^{17}$

Pories et al. ${ }^{16}$ observed that patients with good metabolic response i.e. complete diabetes remission, had a shorter duration of diabetes (1.75 \pm 0.69 vs. $8.79 \pm 2.8$ years).

Schauer ${ }^{17}$ replicated similar findings and showed that patients with a diabetes duration $<5$ years had a greater chance of complete remission after gastric bypass surgery.

Robert et al. ${ }^{18}$ demonstrated that a duration $<4$ years had a $79 \%$ sensitivity and $80 \%$ specificity $(p=0.0001)$ to predict resolution at one year.

In our patients, preoperative BMI and 1-year EWL\% was not a significant factor for remission of diabetes in both groups (Tables $\mathbf{7 , 8}$ ). The role of preoperative BMI in the response to bariatric surgery remains controversial and few authors have demonstrated a link with remission rate. ${ }^{19,20}$ The absence of a significant difference in our study may be attributed to the small group sizes.

Kang et al. ${ }^{19}$ in a meta-analysis study found that a high BMI may be predictive of the success of metabolic surgery for T2DM only in Asian patients. Therefore, it remains uncertain whether BMI is a predictive factor in non-Asian patients. His study also confirmed that disease severity is an important predictive factor of the extent of improvement that can be potentially achieved after bariatric surgery, particularly for Asian patients.

Dixon et al. ${ }^{20}$ observed that patients with a preoperative BMI $>35 \mathrm{~kg} / \mathrm{m} 2$ had higher rate of diabetes remission compared to those with a BMI $<35 \mathrm{~kg} / \mathrm{m} 2$.

Other studies found that a super-obese BMI was not a good predictor of T2DM resolution and may be associated with reduced life expectancy. ${ }^{18}$

Preoperative $\mathrm{HbA1C}$ appears to be predictive for remission of T2DM in our study in both SG and MGB groups (Tables 9,10). This can be explained that poorly controlled diabetic patients will be less sensitive to the improvement in insulin response post-surgery, given the probable pre-existing deficit in secretion by B-cells in the pancreas.

This goes with the study of 127 patients with T2DM, made by Hayes et al. ${ }^{21}$ showing that lower levels of fasting blood glucose and $\mathrm{HbA1c}$ were 
markers for T2DM remission after gastric bypass surgery. Similarly, Robert et al. ${ }^{18}$ concluded that a fasting glucose $<114 \mathrm{mg} / \mathrm{dL}$ and a $\mathrm{HbA} 1 \mathrm{c}<7.1 \%$ were predictors for T2DM resolution at one year, regardless of the type of bariatric operations.

In a study by Jurowich et al. ${ }^{15} 17$ out of 82 participants did not show an improvement in their diabetes with non-responders having a higher preoperative $\mathrm{HbA1c}$ level $(8.34 \%$ vs. $7.78 \%, p=0.033)$.

Our study demonstrated the superiority of MGB over SG in controlling T2DM along 1 year of follow up with lower 1-year HBA1C, FBG, 2HPP blood glucose level in bypass group (total remission rate $(85 \%)$ ) in comparison with SG group (total remission rate (50\%)). (Tables 6-8).

In a study done by Musella et al. ${ }^{22}$ to evaluate the Role of Mini Gastric Bypass/One Anastomosis Gastric Bypass and Sleeve Gastrectomy at 1 Year of follow-up, both surgical procedures achieved T2DM remission if compared to baseline values remission rate from T2DM of $82 / 96$ patients (85.4\%) for those controlled at 1 year, while patients who underwent SG have a remission rate of $67 / 110$ (60.9\%).

Yang et al. ${ }^{23}$ supported the superiority of gastric bypass over sleeve gastrectomy for T2DM treatment. The patients who received MGB achieved a lower $\mathrm{HbA1C}$ and had a lower total cholesterol, triglyceride, and diastolic blood pressure than the patients who received SG.

On the other hand, in the Schauer et al. ${ }^{17}$ study, the diabetes remission rate was similar between MGB and SG groups which do not meet our study.

\section{Conclusion}

MGB is superior to SG in controlling T2DM along 1 year of follow up with lower 1-year HBA1C, FBG, 2HPP blood glucose level in bypass group compared to SG group. Late postoperative complications and long-term maintenance of glycemic control need to be determined by further studies on a larger scale of patients and bigger duration of follow up.

\section{References}

1. Scheen AJ, De Flines J, De Roover A, Paquot N: Bariatric surgery in patients with type 2 diabetes: benefits, risks, indications and perspectives. Diabetes Metab. 2009; 35: 537-543.

2. Schernthaner G, Morton JM: Bariatric surgery in patients with morbid obesity and type 2 diabetes. Diabetes Care. 2008; 31: 297-302.

3. Vetter $M L$, Cardillo $S$, Rickels $M R$, Iqbal $N$ : Narrative review: Effect of bariatric surgery on type 2 diabetes mellitus. Ann Int Med. 2009; 150: 94-103.

4. Schulman AP, Del Genio F, Sinha N, Rubino F: Metabolic surgery for the treatment of type 2 diabetes. Endocr. Pract. 2009; 15: 624-31.

5. Colquitt JL, Picot J, Loveman E, Cleqq AJ: Surgery for obesity. Cochrane Database Syst Rev. 2: CD003641. 2009.

6. Tice JA, Karliner L, Walsh J, Petersen AJ, Feldman MD: Gastric banding or bypass? A systematic review comparing the two most popular bariatric procedures. Am J Med. 2009; 121: 885-893.

7. Kadera BE, Lum K, Grant J, Pryor AD, Portenier DD, DeMaria EJ: Remission of type 2 diabetes after Roux-en-Y gastric bypass is associated with greater weight loss. Surg Obes Relat Dis. 2009; 5: 305-309.

8. Purnell JQ, Flum DR: Bariatric surgery and diabetes: who should be offered the option of remission? JAMA. 2009; 301: 1593-1595.

9. Buchwald $H$, Avidor $Y$, Braunwald $E$, Jensen MD, Pories W, Fahrbach K, Schoelles K: Bariatric surgery: a systematic review and meta-analysis. JAMA. 2014; 292: 1724-1737.

10. Fried $M$, Hainer $V$, Basdevant $A$, Buchwald $H$, Deitel $M$, Finer $\mathrm{N}$ : Interdisciplinary European guidelines for surgery for severe (morbid) obesity. Obes Surg. 2007; 17: 260-270.

11. Kular KS, Manchanda N, Rutledge R: A 6-year experience with 1054 mini-gastric bypasses; First study from Indian subcontinent. Obes Surg. 2014; 24: 1430-1435.

12. Sánchez-Pernaute $A$, Rubio $M A$, Pérez Aguirre E, Barabash A, Cabrerizo L, Torres A: Singleanastomosis duodenoileal bypass with sleeve gastrectomy; metabolic improvement and weight loss in first 100 patients. Surg Obes Relat Dis. 2013; 9(5): 731-5.

13. Still CD, Wood GC, Benotti P, Petrick AT, Gabrielsen J, et al: Preoperative prediction of type 2 diabetes remission after Roux-en-Y gastric bypass surgery: a retrospective cohort study. Lancet Diabetes Endocrinol. 2014; 2: 3845.

14. Huang CK, Shabbir A, Lo CH, Tai CM, Chen YS, Houng JY: Laparoscopic mini gastric bypass for the treatment of type II diabetes mellitus in 
Chinese patients with body mass index of 2535. Obes Surg. 2011; 21(9): 1344-9.

15. Jurowich C, Thalheimer A, Hartmann D, Bender $G$, Seyfried $F$, et al: Improvement of type 2 diabetes mellitus (T2DM) after bariatric surgery-who fails in the early postoperative course? Obes Surg. 2012; 22: 1521-1526.

16. Pories WJ, MacDonald KG, Morgan EJ, Sinha MK, Dohm GL, et al: Surgical treatment of obesity and its effect on diabetes: 10-y follow-up. $\mathbf{A m} \boldsymbol{J}$ Clin Nutr. 1992; 55: 582S-585S.

17. Schauer PR: Effect of laparoscopic Roux-en Y gastric bypass on type 2 diabetes mellitus. Ann Surg. 2003; 238(4): 467-84.

18. Robert M, Ferrand-Gaillard C, Disse E, Espalieu P, Simon C, et al: Predictive factors of type 2 diabetes remission 1 year after bariatric surgery; impact of surgical techniques. Obes Surg. 2013; 23: $770-5$.

19. Kang KC, Shin SH, Lee YJ, Heo YS: Influence of gastrectomy for stomach cancer on type 2 diabetes mellitus for patients with a body mass index less than $30 \mathrm{~kg} / \mathrm{m} 2$. J Korean Surg Soc. 2012; 82(6): 347-55.

20. Dixon JB, Straznicky NE, Lambert EA, Schlaich MP, Lambert GW, et al: Laparoscopic adjustable gastric banding and other devices for the management of obesity. Circulation. 2012; 126(6): 774-85.

21. Hayes MT, Hunt LA, Foo J, Tychinskaya Y, Stubbs RS: A model for predicting the resolution of type 2 diabetes in severely obese subjects following Roux-en Y gastric bypass surgery. Obes Surg. 2011; 21: 910-6.

22. Musella M, Apers J, Rheinwalt $K$, et al: Efficacy of bariatric surgery in type 2 diabetes mellitus remission: the role of mini gastric bypass/ one anastomosis gastric bypass and sleeve gastrectomy at 1 year of follow-up. A European survey. Obesity Surgery. 2015; 26(5): 933-940.

23. Yang $X$, Yang G, Wang W, et al: A meta-analysis: to compare the clinical results between gastric bypass and sleeve gastrectomy for the obese patients. Obes Surg. 2013; 23(7): 1001-10. 